\title{
Aspects of the Phenomenological Theory of Elastic-Plastic Deformation
}

\author{
A. Gupta · D.J. Steigmann · J.S. Stölken
}

Received: 3 September 2010 / Published online: 30 November 2010

(C) The Author(s) 2010. This article is published with open access at Springerlink.com

\begin{abstract}
Several issues pertaining to the phenomenological description of rate-independent plasticity in crystalline solids are discussed. These include energy dissipation, the specification of initial plastic deformation, material symmetry and the modeling of scale-dependent response.
\end{abstract}

Keywords Elasticity $\cdot$ Crystalline solids $\cdot$ Rate-independent plasticity

Mathematics Subject Classification (2000) $74 \mathrm{C} 15 \cdot 74 \mathrm{D} 10 \cdot 74 \mathrm{E} 15$

\section{Introduction}

We are concerned with several open questions in the phenomenological continuum theory of plasticity as it applies to metallic crystals. Among these are the restrictions to be imposed on constitutive equations ensuring that energy dissipation accompanies plastic evolution, the connection between plastic deformation and the crystal lattice and its implications for the initial value of plastic deformation, the issues of scale dependence and plastic spin, the effect of plastic deformation on residual stress, and the role played by a local relaxed configuration that encodes information about the intrinsic properties of the crystal. Attention is confined to rate-independent response in the setting of a purely mechanical theory. We build on our recent efforts to understand and clarify theories based on a factorization of the deformation

Dedicated to the memory of Don Carlson.

\author{
A. Gupta \\ Department of Mechanical Engineering, Indian Institute of Technology, Kanpur, UP 208016, India \\ D.J. Steigmann ( $₫)$ \\ Department of Mechanical Engineering, University of California, Berkeley, CA 94720, USA \\ e-mail: steigman@me.berkeley.edu
}


gradient into elastic and plastic parts. Thus, the present work is a sequel to [1]. The ideas discussed are based on Noll's theory of materially uniform bodies with inhomogeneities [2], the considerable potential of which is amply illustrated by the wide variety of applications discussed in [3, 4] and elsewhere.

We use several standard notational devices, such as $\mathbf{A}^{t}, \mathbf{A}^{-1}, \mathbf{A}^{*}, \operatorname{Sym} \mathbf{A}, \operatorname{Skw} \mathbf{A}$, $\operatorname{Dev} \mathbf{A}, \operatorname{tr} \mathbf{A}$ and $J_{A}$. These are respectively the transpose, the inverse, the cofactor, the symmetric part, the skew part, the deviatoric part, the trace and the determinant of a tensor $\mathbf{A}$, regarded as a linear transformation from a three-dimensional vector space to itself, the latter being identified with the translation space of the usual three-dimensional Euclidean point space. We also use Sym, Skw, Orth and Orth ${ }^{+}$to denote the sets of symmetric, skew, orthogonal and rotation tensors, respectively. We denote the standard tensor product of vectors by interposing the symbol $\otimes$. The inner product of tensors $\mathbf{A}, \mathbf{B}$ is denoted by $\mathbf{A} \cdot \mathbf{B}=\operatorname{tr}\left(\mathbf{A} \mathbf{B}^{t}\right)$, and the associated norm is $|\mathbf{A}|=\sqrt{\mathbf{A} \cdot \mathbf{A}}$. Lastly, the notation $F_{\mathbf{A}}$ stands for the tensor-valued derivative of a scalar-valued function $F(\mathbf{A})$ [5].

Section 2 contains a summary of various relevant equations, including equations of motion and conditions on the strain-energy and yield functions consistent with ideas about the intrinsic properties of a basic crystal type that is deemed to constitute the material. In particular, the yield function is allowed to depend on dislocation density, both to introduce scale effects into the model and to reflect ideas about the nature of yield in crystalline solids. In Sect. 3 we introduce the dissipation and deduce restrictions on the response functions necessary for any process involving plastic flow to be dissipative in the sense that the rate of change of the mechanical energy of an arbitrary part of the body is non-positive. This leads to the conclusion, for conservative boundary-value problems, that the potential energy of the body is likewise non-increasing in such processes for which the initial velocity vanishes and thus minimized by any asymptotically stable equilibria.

Following ideas prevalent in the subject, in Sect. 4 we adapt Il'yushin's postulate [6-9] and examine its consequences, including the maximization of dissipation by stresses belonging to a current yield surface associated with the present value of the dislocation density. There we also discuss the plastic spin and show that it may be suppressed at the constitutive level without affecting the stress, dissipation or motion predicted by the theory. This is important for the determination of the plastic deformation, which in turn is necessary for the specification of the dislocation density. Conversely, plastic spin affects neither elastic strain nor dislocation density, and thus has no effect on the state of the material. These considerations lead, in Sect. 5, to a normality rule giving the plastic deformation rate relative to the current yield surface. The non-standard features of an associated consistency condition, due to dislocation density, are also discussed. In Sect. 6 the role played by plastic deformation in the formulation of the linear theory of elasticity for residually-stressed solids is explored. Simple formulas are given connecting the residual stress and the stiffness to the intrinsic properties of the underlying crystal. The model is specialized to cubic crystals in Sect. 7, where the forms assumed by the strain-energy and yield functions are discussed and a formula for the effect of dislocation on yield is proposed.

\section{Outline of the Basic Theory}

The variables that describe the evolution of an elastic-plastic solid in the purely mechanical theory are the motion $\chi(\mathbf{X}, t)$ and the plastic deformation tensor $\mathbf{K}(\mathbf{X}, t)$, where $\mathbf{X}$ is the position of a material point in a fixed reference placement $\kappa_{r}$ of the body. The values $\mathbf{x}=\chi(\mathbf{X}, t)$ are the positions of these points at time $t$ and generate the current placement 
$\kappa_{t}$ of the body as $\mathbf{X}$ ranges over $\kappa_{r}$. As is usual, we suppose positions in $\kappa_{r}$ and $\kappa_{t}$ to be in one-to-one correspondence and thus the deformation gradient, $\mathbf{F}=\nabla \boldsymbol{\chi}$, to be invertible. These variables are used to define the elastic deformation

$$
\mathbf{H}=\mathbf{F K} \text {. }
$$

The measure of plastic deformation used most frequently in the literature is

$$
\mathbf{G}=\mathbf{K}^{-1}
$$

We further suppose $\kappa_{r}$ to be a kinematically possible configuration of the body, so that $J_{F}>0$. The elastic deformation is assumed to be the value of the deformation gradient in the absence of plastic deformation, this condition being defined by $\mathbf{K}=\mathbf{I}$, the identity for second-order tensors. Thus, we impose $J_{H}>0$ and conclude that $J_{K}>0$. The elastic strain energy of the body is

$$
U=\int_{\kappa_{t}} \psi(\mathbf{H}) d v
$$

where $\psi$ is the energy per unit volume of $\kappa_{t}$. We assume the material of the body to be uniform in the sense that the strain-energy function does not depend explicitly on $\mathbf{X}$.

The local equations of motion are

$$
\operatorname{Div} \mathbf{P}+\rho_{r} \mathbf{b}=\rho_{r} \ddot{\mathbf{x}}, \quad \mathbf{P F}^{t} \in \operatorname{Sym} \quad \text { in } \kappa_{r},
$$

where $\rho_{r}(\mathbf{X})$ is the mass density in $\kappa_{r}$, Div is the referential divergence (i.e., the divergence with respect to $\mathbf{X})$, superposed dots are used to denote material derivatives $(\partial / \partial t$ at fixed $\mathbf{X}), \mathbf{b}$ is the body force per unit mass, and

$$
\mathbf{P}=\mathbf{F} \Pi
$$

is the Piola stress, in which

$$
\Pi=J_{K}^{-1} \mathbf{K S K}^{t}
$$

is the 2nd Piola-Kirchhoff stress relative to $\kappa_{r}$, and $\mathbf{S}=\hat{\mathbf{S}}(\mathbf{C})$, where

$$
\hat{\mathbf{S}}(\mathbf{C})=2 \hat{W}_{\mathbf{C}}
$$

is that relative to an intermediate configuration $\kappa_{i}$ in which $\mathbf{H}=\mathbf{I}$. The strain-energy function referred to $\kappa_{i}$ is

$$
W(\mathbf{H})=J_{H} \psi(\mathbf{H}),
$$

and, in (7), $\hat{W}$ is the reduced strain energy defined by

$$
\hat{W}(\mathbf{C})=W(\mathbf{H}),
$$

where

$$
\mathbf{C}=\mathbf{H}^{t} \mathbf{H}
$$

is the elastic Cauchy-Green deformation tensor. That the strain energy should depend on $\mathbf{H}$ via $\mathbf{C}$ is necessary and sufficient for the symmetry of the Cauchy stress $\mathbf{T}$, related to $\mathbf{P}$ by 
$\mathbf{P}=\mathbf{T F}^{*}$ (see $[1,10]$ ). This, of course, is equivalent to $(4)_{2}$. The Cauchy stress is given in terms of $\mathbf{S}$ by

$$
J_{H} \mathbf{T}=\mathbf{H S H}^{t} .
$$

The strain-energy function is subject to the restriction

$$
\hat{W}(\mathbf{C})=\hat{W}\left(\mathbf{R}^{t} \mathbf{C R}\right)
$$

associated with the transformation $\mathbf{H} \rightarrow \mathbf{H R}$, where the tensor $\mathbf{R} \in \mathrm{Orth}^{+}$is an arbitrary element of the symmetry group for the material; i.e., the symmetry group relative to the elastically undistorted configuration $\kappa_{i}$ (see $[1,4]$ for further discussion). In particular, material uniformity implies that this restriction is effective, with the same symmetry group, at every material point. Using (7), it is straightforward to show that

$$
\mathbf{S} \rightarrow \mathbf{R}^{t} \mathbf{S R}
$$

under symmetry transformations and hence that $\mathbf{T}$ is invariant.

The system (4) is augmented by suitable boundary and initial data, the former typically being of the type:

$$
\chi(\mathbf{X}, t) \text { and } \mathbf{p}(\mathbf{X}, t) \text { assigned on }\left(\partial \kappa_{r}\right)_{\chi} \text { and }\left(\partial \kappa_{r}\right)_{p} \text {, respectively, }
$$

where $\left(\partial \kappa_{r}\right)_{\chi}$ and $\left(\partial \kappa_{r}\right)_{p}$ are complementary parts of the boundary $\partial \kappa_{r}$ of $\kappa_{r}$, and

$$
\mathbf{p}=\mathbf{P N}
$$

is the traction, $\mathbf{N}$ being the exterior unit normal to $\partial \kappa_{r}$. Initial data

$$
\chi\left(\mathbf{X}, t_{0}\right)=\chi_{0}(\mathbf{X}), \quad \dot{\chi}\left(\mathbf{X}, t_{0}\right)=\dot{\chi}_{0}(\mathbf{X}), \quad \mathbf{K}\left(\mathbf{X}, t_{0}\right)=\mathbf{K}_{0}(\mathbf{X})
$$

are imposed on the closure of $\kappa_{r}$, these being compatible with (14) at $t=t_{0}$. A flow rule for the evolution of $\mathbf{K}$ is discussed in Sect. 5.

Constitutive Hypothesis and the Intermediate Configuration

Following our earlier work [1], we suppose $\hat{W}(\cdot)$ to be a strictly convex function with a minimum at $\mathbf{C}=\mathbf{I}$. This restriction is appropriate for metallic crystals in the elastic range; i.e., for $\mathbf{C}$ close to $\mathbf{I}$. It implies, for a given motion $\chi$ and Cauchy stress $\mathbf{T}$, that the intermediate configuration $\kappa_{i}$ is determined at any fixed instant $t$, apart from orientation, by an equilibrium unloading process to a state of zero stress [1]. This process can be achieved in principle [1], if only locally, and is independent of the processes that the present theory purports to describe. Thus, if, at fixed $t, \mathbf{H}_{1}$ and $\mathbf{H}_{2}$ are elastic deformations to (the translation space of) $\kappa_{t}$ from local configurations $\kappa_{i_{1}}$ and $\kappa_{i_{2}}$, respectively, then there is a rotation $\mathbf{A}$ such that

$$
\mathbf{H}_{1}=\mathbf{H}_{2} \mathbf{A} \text {. }
$$

Let $W_{1}\left(\mathbf{H}_{1}\right)$ be the strain-energy function associated with $\kappa_{i_{1}}$. The Cauchy stress obtained by using $\kappa_{i_{2}}$ coincides with that derived by using $\kappa_{i_{1}}$, as assumed, if and only if the strainenergy function associated with it is $W_{2}\left(\mathbf{H}_{2}\right)=W_{1}\left(\mathbf{H}_{2} \mathbf{A}\right)$ [1]. The rotation may be timedependent, but is held fixed when relating the strain-energy and stress-response functions 
pertaining to two local references in accord with the aforementioned unloading process, performed at fixed time. In other words, at each instant elastic unloading to a state of zero stress yields infinitely many local relaxed configurations that are related to each other by rotations, these being associated with the considered instant and thus independent of time. Equation (7) then yields the relation between the stresses relative to $\kappa_{i_{1}}$ and $\kappa_{i_{2}}$ as

$$
\mathbf{S}_{1}=\mathbf{A}^{t} \mathbf{S}_{2} \mathbf{A}
$$

\section{Lattice Vectors and the Initial Plastic Deformation}

The theory described here is applicable to single crystals. In conventional crystal-elasticity theory, fixed linearly independent lattice vectors $\mathbf{l}_{i}(i \in\{1,2,3\})$ are mapped to their images $\mathbf{t}_{i}$ in $\kappa_{t}$ in accordance with the Cauchy-Born hypothesis. That is, they are regarded as material vectors. Here, we retain this hypothesis insofar as the elastic deformation is concerned. Thus, we suppose that $\mathbf{t}_{i}=\mathbf{H l}_{i}$ where $\mathbf{l}_{j}$ are the lattice vectors in $\kappa_{i}$. Indeed, we identify $\kappa_{i}$ with $\operatorname{Span}\left\{\mathbf{I}_{1}, \mathbf{l}_{2}, \mathbf{l}_{3}\right\}$. The $\mathbf{t}_{i}$ are observable in principle. In practice they are computed from their duals $\mathbf{t}^{i}$, which are measured in X-Ray diffraction experiments [11].

It follows from (1) that $\mathbf{t}_{i}=\mathbf{F} \mathbf{r}_{i}$, where $\mathbf{r}_{i}=\mathbf{K} \mathbf{l}_{i}$ are the lattice vectors in $\kappa_{r}$. The plastic deformation is then given by $\mathbf{K}=\mathbf{r}_{i} \otimes \mathbf{l}^{i}$, where the $\mathbf{l}^{j}$ are the duals of the $\mathbf{l}_{j}$. To see this we write $\mathbf{K}=\mathbf{K I}$ with $\mathbf{I}=\mathbf{l}_{i} \otimes \mathbf{l}^{i}$. It follows similarly that $\mathbf{H}=\mathbf{t}_{i} \otimes \mathbf{l}^{i}$. We assume the $\mathbf{l}_{i}$ associated with a material point to be fixed once and for all and thus to be material vectors. These are the lattice vectors of the unstressed, undistorted lattice. The orientation of the associated triad may be chosen arbitrarily. However, in view of our assumption of material uniformity, once selected these vectors are extended to every material point and are therefore independent of $\mathbf{X}$. Stated differently, the set of lattice vectors is assumed to be an assigned property of the uniform material. Accordingly, $\dot{\mathbf{K}}(X, t)=\dot{\mathbf{r}}_{i}(\mathbf{X}, t) \otimes \mathbf{l}^{i}$. We may thus interpret plastic flow as the failure of the $\mathbf{r}_{i}$ to be material vectors. Further, the initial value $\mathbf{K}_{0}(\mathbf{X})$ in (16) is given by $\mathbf{r}_{i}^{0}(\mathbf{X}) \otimes \mathbf{l}^{i}$, where $\mathbf{r}_{i}^{0}$ are the values of $\mathbf{r}_{i}$ at time $t_{0}$. If $\kappa_{r}$ is identified with $\kappa_{t_{0}}$, then these can be computed from their measurable duals.

\section{Yield Criterion}

Following conventional ideas for the description of rate-independent response we assume plastic flow to be possible only if the material is in a state of yield. We express this as the requirement that the elastic deformation belong to a manifold that may be parametrized by other variables. For example, motivated by G.I. Taylor's formula [12] giving the flow stress as a function of dislocation density, and using the fact that the stress $\mathbf{S}$ may be expressed in terms of $\mathbf{C}$ via (7), we assume yield to be possible if [1]

$$
G(\mathbf{E}, \boldsymbol{\alpha})=0,
$$

where $G$ is a suitable yield function,

$$
\mathbf{E}=\frac{1}{2}(\mathbf{C}-\mathbf{I})
$$

is the elastic strain and

$$
\boldsymbol{\alpha}=J_{K} \mathbf{K}^{-1} \operatorname{Curl} \mathbf{K}^{-1}
$$

is the dislocation density. Relevant to the ensuing development is the current yield surface, defined, for fixed $\boldsymbol{\alpha}$, by $G(\cdot, \boldsymbol{\alpha})=0$. For simplicity's sake we assume $G$ to be differentiable. 
In (21), Curl is the referential curl operation defined in terms of the usual vector operation [5] by

$$
(\operatorname{Curl} \mathbf{A}) \mathbf{c}=\operatorname{Curl}\left(\mathbf{A}^{t} \mathbf{c}\right)
$$

for any fixed vector $\mathbf{c}$.

Plastic evolution; i.e., $\dot{\mathbf{K}} \neq \mathbf{0}$, is deemed to be possible only when (19) is satisfied, and the variables $\mathbf{E}, \boldsymbol{\alpha}$ are constrained to belong to the elastic range defined by $G \leq 0$, assumed to be a connected set in $\mathbb{R}^{6} \times \mathbb{R}^{9}$. In view of our restriction to materially uniform bodies we require that the same yield function pertain to all material points.

In [1] it is shown that (19) is invariant under superposed rigid-body motions and (global) changes of reference placement and is thus intrinsic to the material, provided that the function $G$ is likewise invariant (see also $[13,14]$ ). The same statement applies to the reduced strain-energy function (9) and to the associated stress, defined by (7). In particular, the stated invariance properties are possessed by the tensors $\mathbf{C}$ and $\boldsymbol{\alpha}[1,2,13,14]$. Further, the yield function is subject to the same material-symmetry restriction as that imposed on the strainenergy function; i.e.,

$$
G(\mathbf{E}, \boldsymbol{\alpha})=G\left(\mathbf{R}^{t} \mathbf{E R}, \mathbf{R}^{t} \boldsymbol{\alpha} \mathbf{R}\right) .
$$

It is important to note that the dislocation density is well-defined under symmetry transformations only if the symmetry group is discrete (see Theorem 8 of [2]). Accordingly, yield functions of the kind considered are meaningful only for crystalline solids.

The body is dislocated if $\boldsymbol{\alpha}$ does not vanish; in this case $\mathbf{K}^{-1}$ is not a gradient and from (1) it follows that neither is $\mathbf{H}$. In fact [1],

$$
\boldsymbol{\alpha}=J_{H} \mathbf{H}^{-1} \operatorname{curl} \mathbf{H}^{-1},
$$

in which curl is the spatial curl. Then, $\kappa_{i}$ has only local significance in the sense that it cannot be identified with a global placement of the body in Euclidean space. That is, a differentiable position field that identifies material points in $\kappa_{i}$ does not exist.

In dislocation theory $\alpha$ is frequently referred to as the density of geometrically necessary dislocations. Dislocations not accounted for by $\alpha$ are said to be statistically stored [15]. The distinction is due to the implicit choice of length scale at which the theory is intended to apply. In particular, if material points in the continuum theory correspond to many unit cells of the crystal lattice, then there may be dislocations existing below the scale of a material point that are not accounted for by the density $\boldsymbol{\alpha}$. These are classified as statistically stored. They are not of the geometrically necessary variety because they occur either as dipoles or as closed loops or self-terminating structures whose largest dimensions are smaller than the smallest scale resolved by the considered continuum theory. These make no net contribution to Burgers circuits $[1,14]$ in the continuum theory and so are not accounted for by $\boldsymbol{\alpha}$. Consequently, the classification of dislocations is inherently scale dependent. If the continuum theory is applicable at arbitrarily small length scales, then the associated density of statistically stored dislocations vanishes. This view is implicit in Taylor's considerations [12]. On the other hand, if the theory is designed for use at larger scales, then presumably the associated statistically stored dislocations should play a role in the constitutive equations for yield and plastic flow. One possibility is to regard them as internal variables. They could then affect yielding and evolve according to suitable flow rules. Although a number of proposals for associated constitutive equations have been advanced, it appears to us that the issue is far from settled. However, a comprehensive theory of plasticity with (generic) internal variables, compatible with the present framework, has been developed elsewhere $[16,17]$. 
We do not consider statistically stored dislocations in this work. Instead, we concentrate on the non-standard features of the theory due to the presence of the density defined by (21) in yield criteria. In particular, we note that the presence of the dislocation density in the yield function introduces a natural length scale in the theory. Scale effects have been documented in experiments [18] and their role in relevant boundary-value problems for elastic-plastic materials has been studied [19, 20]. Thus, (19) furnishes an avenue for the description of such effects regardless of its efficacy or otherwise as a comprehensive model of the role played by dislocations per se in the plastic response of metals. Indeed, assuming such effects to be attributable to the plastic deformation and its gradient, they necessarily manifest themselves in constitutive equations through the tensor $\boldsymbol{\alpha}[13,14]$.

\section{Energy Dissipation and Asymptotically Stable Equilibria}

The power of the forces acting on an arbitrary part $p$ of the body is

$$
P(\pi, t)=\int_{\partial \pi} \mathbf{p} \cdot \dot{\mathbf{x}} d A+\int_{\pi} \rho_{r} \mathbf{b} \cdot \dot{\mathbf{x}} d V,
$$

where $\pi$, with piecewise smooth boundary $\partial \pi$, is the region occupied by $p$ in the reference configuration $\kappa_{r}$. The sum of the kinetic and strain energies of $p$ is

$$
\int_{\pi} \Phi d V, \quad \text { where } \Phi=\Psi+\frac{1}{2} \rho_{r}|\dot{\mathbf{x}}|^{2}
$$

and

$$
\Psi(\mathbf{F}, \mathbf{K})=J_{K} W(\mathbf{F K})
$$

is the strain energy per unit volume of $\kappa_{r}$. The dissipation is denoted by $\mathcal{D}(\pi, t)$ and defined as the difference between the power supplied to $p$ and the rate of change of the total energy in $p$. Thus,

$$
\mathcal{D}(\pi, t)=P(\pi, t)-\frac{d}{d t} \int_{\pi} \Phi d V
$$

The dissipation is assumed to be non-negative for every sub-body; i.e.,

$$
\mathcal{D}(\pi, t) \geq 0 .
$$

We are interested in potential applications of the theory to shock physics. Let $s \subset \kappa_{r}$ be a smooth shock surface propagating with speed $U$ in the direction of its local orientation $\mathbf{N}_{s}$. Associated jump conditions holding on such singular surfaces are well known [21]. Thus,

$$
[\mathbf{P}] \mathbf{N}_{s}=-\rho_{r} U[\dot{\mathbf{x}}] \quad \text { and } \quad[\dot{\mathbf{x}}] \otimes \mathbf{N}_{s}=-U[\mathbf{F}] \quad \text { on } s .
$$

Here, $[\cdot]=(\cdot)^{+}-(\cdot)^{-}$is the discontinuity on $s$, where superscripts ${ }^{ \pm}$are used to denote the limits of functions as $s$ is approached from the regions into which $\mathbf{N}_{s}$ and $-\mathbf{N}_{s}$ are directed, respectively. The average of these limits is denoted by $\langle\cdot\rangle$. Thus, $\langle\cdot\rangle=\frac{1}{2}\left[(\cdot)^{+}+(\cdot)^{-}\right]$and it follows immediately that $[f g]=[f]\langle g\rangle+\langle f\rangle[g]$.

Regarding the balance (28), we have [21]

$$
\frac{d}{d t} \int_{\pi} \Phi d V=\int_{\pi} \dot{\Phi} d V-\int_{s^{\prime}} U[\Phi] d A,
$$


where $s^{\prime}=s \cap \pi$. Using (15) and applying the divergence theorem to the subregions of smoothness comprising $\pi$ yields

$$
\int_{\partial \pi} \mathbf{p} \cdot \dot{\mathbf{x}} d A=\int_{\pi} \operatorname{Div}\left(\mathbf{P}^{t} \dot{\mathbf{x}}\right) d V+\int_{S^{\prime}}\left[\mathbf{P}^{t} \dot{\mathbf{x}}\right] \cdot \mathbf{N}_{s} d A .
$$

Next, we form the inner product of the material velocity with the local equation of motion (4) 1 holding in $\pi \backslash s$, obtaining

$$
\operatorname{Div}\left(\mathbf{P}^{t} \dot{\mathbf{x}}\right)+\rho_{r} \mathbf{b} \cdot \dot{\mathbf{x}}=\left(\frac{1}{2} \rho_{r}|\dot{\mathbf{x}}|^{2}\right)+\mathbf{P} \cdot \dot{\mathbf{F}}=\dot{\Phi}+D,
$$

where [1]

$$
D=\mathcal{E} \cdot \dot{\mathbf{K}} \mathbf{K}^{-1}
$$

in which

$$
\mathcal{E}=\Psi \mathbf{I}-\mathbf{F}^{t} \mathbf{P}
$$

is Eshelby's energy-momentum tensor.

The contribution of the integral over $s$ may be reduced with the aid of the identities

$$
\left[\mathbf{P}^{t} \dot{\mathbf{x}}\right] \cdot \mathbf{N}_{s}=\left(\left[\mathbf{P}^{t}\right]\langle\dot{\mathbf{x}}\rangle+\left\langle\mathbf{P}^{t}\right\rangle[\dot{\mathbf{x}}]\right) \cdot \mathbf{N}_{s}=\left[\mathbf{P} \mathbf{N}_{s}\right] \cdot\langle\dot{\mathbf{x}}\rangle+\langle\mathbf{P}\rangle \cdot[\dot{\mathbf{x}}] \otimes \mathbf{N}_{s},
$$

which may be combined with the shock relation $(30)_{1}$ and $[\dot{\mathbf{x}}] \cdot\langle\dot{\mathbf{x}}\rangle=\frac{1}{2}\left[|\dot{\mathbf{x}}|^{2}\right]$ to derive

$$
\left[\mathbf{P}^{t} \dot{\mathbf{x}}\right] \cdot \mathbf{N}_{s}=-\frac{1}{2} \rho_{r}\left[|\dot{\mathbf{x}}|^{2}\right]-U\langle\mathbf{P}\rangle \cdot[\mathbf{F}]
$$

This is further reduced with the aid of (30) and $[\mathbf{F}]=[\mathbf{F}] \mathbf{N}_{s} \otimes \mathbf{N}_{s}$. Thus,

$$
\langle\mathbf{P}\rangle \cdot[\mathbf{F}]=[\mathbf{F}] \mathbf{N}_{s} \cdot\langle\mathbf{P}\rangle \mathbf{N}_{s}=\mathbf{N}_{s} \cdot\left[\mathbf{F}^{t}\right]\langle\mathbf{P}\rangle \mathbf{N}_{s}=\mathbf{N}_{s} \cdot\left[\mathbf{F}^{t} \mathbf{P}\right] \mathbf{N}_{s}-\rho_{r} U^{2} \mathbf{N}_{s} \cdot\left\langle\mathbf{F}^{t}\right\rangle[\mathbf{F}] \mathbf{N}_{s} .
$$

In the final term we use $\mathbf{N}_{s} \cdot\left\langle\mathbf{F}^{t}\right\rangle[\mathbf{F}] \mathbf{N}_{s}=\mathbf{N}_{s} \cdot[\mathbf{F}]\left\langle\mathbf{F}^{t}\right\rangle \mathbf{N}_{s}=\frac{1}{2} \mathbf{N}_{s} \cdot\left[\mathbf{F}^{t} \mathbf{F}\right] \mathbf{N}_{s}$ and combine the resulting expression with (25), (26), (28), (32) and (37), obtaining

$$
\mathcal{D}(\pi, t)=\int_{\pi} D d V+\int_{s^{\prime}} D_{s} d A
$$

where $D$ is defined by (34), and

$$
D_{s}=U \mathbf{N}_{s} \cdot\left([\mathcal{E}]+\frac{1}{2} \rho_{r} U^{2}\left[\mathbf{F}^{t} \mathbf{F}\right]\right) \mathbf{N}_{s} .
$$

Equation (39) applies to a subset $\pi^{\prime}$ of $\pi$ containing no discontinuities; i.e.,

$$
\mathcal{D}\left(\pi^{\prime}, t\right)=\int_{\pi^{\prime}} D d V \geq 0 .
$$

Because $\pi^{\prime}$ is otherwise arbitrary, we have

$$
D \geq 0 \quad \text { a.e. } \quad \text { in } \kappa .
$$


Application of (39) to a region $\pi^{\prime}$ intersecting $s$, followed by collapse of $\pi^{\prime}$ onto $s$, furnishes

$$
\mathcal{D}\left(\pi^{\prime}, t\right)=\int_{s^{\prime \prime}} D_{s} d A \geq 0
$$

where $s^{\prime \prime}=\pi^{\prime} \cap s$. Localization now yields

$$
D_{s} \geq 0 \quad \text { on } s .
$$

(see also [22]). Conversely, (42) and (44) yield (29).

It is immediate that (42) holds as an equality if $\mathbf{K}=\mathbf{0}$. Here, we assume that (42) holds as an equality if and only if $\dot{\mathbf{K}}=\mathbf{0}$. Then,

$$
D>0 \text { if and only if } \dot{\mathbf{K}} \neq \mathbf{0} \text { a.e. in } \kappa .
$$

This is the hypothesis of strong dissipation introduced in [1], according to which plastic evolution is inherently dissipative. It is shown there to imply that $\mathbf{K}$ is invariant under superposed rigid-body motions.

We apply (28) to the special case $\pi=\kappa$ for which the traction $\mathbf{p}$ is assigned on a part $\partial \kappa_{p}$ of the boundary and position is assigned on the complementary part. In the case of conservative loading the body force in $\kappa$ and traction on $\partial \kappa_{p}$ are such that

$$
P(\kappa, t)=\frac{d}{d t} L,
$$

where $L$ is a suitable load potential. It follows that

$$
\frac{d}{d t}[K(\kappa, t)+E(\kappa, t)]+\mathcal{D}(\kappa, t)=0
$$

where

$$
E=U-L
$$

is the total potential energy. Inequality (29) then implies that the mechanical energy $K+$ $E$ of the body is dissipated. Further, (45) implies that the dissipation is strict at any time when $\dot{\mathbf{K}}$ does not vanish at some point of the body. Accordingly, if $\{\chi(\mathbf{X}, t), \mathbf{K}(\mathbf{X}, t)\}$, with $\dot{\chi}\left(\mathbf{X}, t_{0}\right)=\mathbf{0}$, is a trajectory tending to $\left\{\chi_{\infty}(\mathbf{X}), \mathbf{K}_{\infty}(\mathbf{X})\right\}$ a.e. as $t \rightarrow \infty$, then $E_{\infty} \leq E_{0}$, where $E_{0}$ and $E_{\infty}$ are the values of $E$ at times $t_{0}$ and infinity. It follows that asymptotically stable states minimize the potential energy of the body.

\section{I'llyushin's Postulate and Its Consequences}

I'llyushin's postulate is the requirement that the net work performed on an arbitrary subbody be non-negative in every closed cycle of deformation [6-9]. Here, 'closed' refers to the restriction that deformations, as well as velocities, coincide at times $t_{1}$ and $t_{2}$ [5]; in particular, $\chi\left(\cdot, t_{1}\right)=\chi\left(\cdot, t_{2}\right)$ and $\mathbf{F}\left(t_{1}\right)=\mathbf{F}\left(t_{2}\right)$. Using (25), (28), (31)-(33) and the localization theorem [5], we derive from this postulate the inequality

$$
\int_{t_{1}}^{t_{2}} \mathbf{P} \cdot \dot{\mathbf{F}} d t \geq 0
$$


holding at every material point in the interior of the body not located on a singular surface. Equations (26) and (33) are then used to obtain

$$
\int_{t_{1}}^{t_{2}}(\dot{\Psi}+D) d t \geq 0
$$

Suppose plastic evolution occurs in the prescribed interval $\left[t_{a}, t_{b}\right] \subset\left[t_{1}, t_{2}\right]$. Thus, $\dot{\mathbf{K}}$ and the dissipation are non-vanishing only for some $t \in\left[t_{a}, t_{b}\right]$. In particular, $\mathbf{K}\left(t_{1,2}\right)=\mathbf{K}\left(t_{a, b}\right)$, respectively. Inequality (50) becomes

$$
\Psi\left(\mathbf{F}\left(t_{1}\right), \mathbf{K}\left(t_{b}\right)\right)-\Psi\left(\mathbf{F}\left(t_{1}\right), \mathbf{K}\left(t_{a}\right)\right)+\int_{t_{a}}^{t_{b}} D d t \geq 0,
$$

where we have used $\mathbf{F}\left(t_{1}\right)=\mathbf{F}\left(t_{2}\right)$. This may be recast as

$$
\int_{t_{a}}^{t_{b}}\left[\Psi_{\mathbf{K}}\left(\mathbf{F}\left(t_{1}\right), \mathbf{K}(t)\right) \cdot \dot{\mathbf{K}}(t)+D(t)\right] d t \geq 0,
$$

where the derivative in the first term is calculated at fixed $\mathbf{F}$. Dividing by $t_{b}-t_{a}$, using the mean-value theorem, and passing to the limit $t_{b} \rightarrow t_{a}$ yields

$$
\Psi_{\mathbf{K}}\left(\mathbf{F}\left(t_{1}\right), \mathbf{K}\left(t_{a}\right)\right) \cdot \dot{\mathbf{K}}\left(t_{a}\right)+D\left(t_{a}\right) \geq 0 .
$$

From (27) it is straightforward to show [1] that

$$
\Psi_{\mathbf{K}}\left(\mathbf{F}\left(t_{1}\right), \mathbf{K}(t)\right)=-\mathcal{E}\left(\mathbf{F}\left(t_{1}\right), \mathbf{K}(t)\right) \mathbf{K}(t)^{-t},
$$

where $\mathcal{E}$ is defined by (35). Combining this with (34) we reduce (53) to

$$
\left[\mathcal{E}\left(\mathbf{F}\left(t_{a}\right), \mathbf{K}\left(t_{a}\right)\right)-\mathcal{E}\left(\mathbf{F}\left(t_{1}\right), \mathbf{K}\left(t_{a}\right)\right)\right] \cdot \dot{\mathbf{K}}\left(t_{a}\right) \mathbf{K}\left(t_{a}\right)^{-1} \geq 0 .
$$

This is a restriction on the state which, at time $t_{1}$, lies within the elastic range that is current at time $t_{a}$. For, (55) involves the plastic deformation only through $\mathbf{K}\left(t_{a}\right)$; the associated dislocation density is then fixed at the value $\boldsymbol{\alpha}\left(t_{a}\right)$ defined by (21). Further, (55) implies that the dissipation is maximized at states that lie on the current yield surface.

We note that (55) is equivalent to

$$
\left[\mathcal{E}^{\prime}\left(\mathbf{C}\left(t_{a}\right)\right)-\mathcal{E}^{\prime}\left(\mathbf{C}\left(t_{1}\right)\right)\right] \cdot \mathbf{K}\left(t_{a}\right)^{-1} \dot{\mathbf{K}}\left(t_{a}\right) \geq 0,
$$

where [1]

$$
\mathcal{E}^{\prime}=\hat{W}(\mathbf{C}) \mathbf{I}-\mathbf{C} \hat{\mathbf{S}}(\mathbf{C})
$$

is the push-forward to $\kappa_{i}$ of the Eshelby tensor, defined by

$$
\mathcal{E}^{\prime}=J_{K} \mathbf{K}^{t} \mathcal{E} \mathbf{K}^{-t} .
$$

Small Elastic Strain

In view of our interest in metallic crystals, it is appropriate to restrict the elastic strain to be small. Accordingly, the strain energy and stress are approximated by

$$
\hat{W} \simeq \tilde{W}(\mathbf{E})=\frac{1}{2} \mathbf{E} \cdot \mathcal{C}[\mathbf{E}] \quad \text { and } \quad \mathbf{S} \simeq \tilde{\mathbf{S}}(\mathbf{E})=\mathcal{C}[\mathbf{E}],
$$


respectively, where $\mathcal{C}$ is the fixed fourth-order tensor of elastic moduli pertaining to the undistorted crystal, possessing the usual major and minor symmetries. The convexity hypothesis of Sect. 2 implies that $\mathcal{C}$ is positive definite on the linear space of symmetric tensors. From (20) and (57), $\mathcal{E}^{\prime}=-\mathbf{S}+o(|\mathbf{E}|)$, and, to leading order, (56) reduces to

$$
\left[\mathbf{S}\left(t_{a}\right)-\mathbf{S}\left(t_{1}\right)\right] \cdot \dot{\mathbf{G}}\left(t_{a}\right) \mathbf{G}\left(t_{a}\right)^{-1} \geq 0,
$$

where $\mathbf{S}(t)=\tilde{\mathbf{S}}(\mathbf{E}(t))$ and $\mathbf{E}\left(t_{1}\right)$ is such that $G\left(\mathbf{E}\left(t_{1}\right), \boldsymbol{\alpha}\left(t_{a}\right)\right) \leq 0$; i.e., the elastic deformation at the start of the cycle belongs to the elastic range that is current at time $t_{a}$. Our constitutive hypothesis also implies that $\tilde{\mathbf{S}}(\mathbf{E})$ is invertible, and hence that the yield function may be expressed in terms of $\mathbf{S}$ instead of $\mathbf{E}$. We write

$$
F(\mathbf{S}, \boldsymbol{\alpha})=G(\tilde{\mathbf{E}}(\mathbf{S}), \boldsymbol{\alpha}),
$$

where $\tilde{\mathbf{E}}(\cdot)$ is the inverse of the function $\tilde{\mathbf{S}}(\cdot)$. The current yield function is

$$
H(\cdot)=F(\cdot, \boldsymbol{\alpha})
$$

and the current elastic range is defined by $H \leq 0$.

To the same order in elastic strain, the dissipation inequality (42) is reduced, with the aid of (34) and (58), to the statement

$$
\mathbf{S} \cdot \dot{\mathbf{G}} \mathbf{G}^{-1} \geq 0 \text { for all } \dot{\mathbf{G}},
$$

and the strong dissipation hypothesis (45) reduces to

$$
\mathbf{S} \cdot \dot{\mathbf{G}} \mathbf{G}^{-1}>0 \text { if and only if } \dot{\mathbf{G}} \neq \mathbf{0} .
$$

Accordingly, the plastic spin, $\mathrm{Skw} \dot{\mathbf{G}} \mathbf{G}^{-1}$, plays no role in dissipation. Thus, to simplify matters, we assume that

$$
\dot{\mathbf{G}} \mathbf{G}^{-1} \in \mathrm{Sym} .
$$

Irrelevance of Plastic Spin

We emphasize the fact that the elastic deformation, being defined in terms of a restriction on the stress (Sect. 2), is simultaneously both kinematical and constitutive in character. Our hypothesis of strong dissipation implies the same for the plastic deformation; that is, plastic deformation is here defined, in part, in terms of a restriction on material response. This contrasts with conventional ideas in continuum mechanics, according to which kinematical and dynamical variables have meaning in their own right, independent of any constitutive framework. In [1] our interpretation is exploited to justify the imposition of (65) as a constitutive hypothesis. That is, plastic spin plays no role in the storage or dissipation of energy by the material, and so material properties are deemed to be insensitive to it. Accordingly, as far as constitutive behavior is concerned, (65) may be imposed without loss of generality.

To elaborate, consider the dissipations relative to two local configurations $\kappa_{i_{1}}$ and $\kappa_{i_{2}}$ related by a time-dependent rotation $\mathbf{A}(t)$ for $t$ belonging to an open interval $\mathcal{I}$. At each fixed $t \in \mathcal{I}$, we invoke the unloading process discussed in Sect. 2 to conclude that both local configurations deliver the same deformation and Cauchy stress, provided that $\mathbf{G}_{2}=\mathbf{A G}_{1}$ at that-hence every-instant in $\mathcal{I}$. Thus, differentiation with respect to time is meaningful and yields

$$
\dot{\mathbf{G}}_{2} \mathbf{G}_{2}^{-1}=\mathbf{A}\left(\dot{\mathbf{G}}_{1} \mathbf{G}_{1}^{-1}+\mathbf{A}^{t} \dot{\mathbf{A}}\right) \mathbf{A}^{t}
$$


Accordingly, from (18), applied at some $t \in \mathcal{I}$,

$$
\mathbf{S}_{1} \cdot \dot{\mathbf{G}}_{1} \mathbf{G}_{1}^{-1}=\mathbf{S}_{2} \cdot \dot{\mathbf{G}}_{2} \mathbf{G}_{2}^{-1},
$$

and the dissipations relative to the two local configurations coincide. The same statement applies to the exact expression for the dissipation given by (34). Further, the dislocation density remains invariant modulo rotation [1]; i.e., $\boldsymbol{\alpha}_{1}=\mathbf{A}^{t} \boldsymbol{\alpha}_{2} \mathbf{A}$, and the rule $G_{1}\left(\mathbf{E}_{1}, \boldsymbol{\alpha}_{1}\right)=G_{2}\left(\mathbf{E}_{2}\right.$, $\boldsymbol{\alpha}_{2}$ ) ensures that the two local configurations furnish the same predictions with respect to yield.

We now impose (65) on $\kappa_{i_{2}}$; i.e., we require that $\dot{\mathbf{G}}_{2} \mathbf{G}_{2}^{-1} \in \operatorname{Sym}$ and set $\mathbf{W}(t)=$ Skw $\dot{\mathbf{G}}_{1} \mathbf{G}_{1}^{-1}$ in (66), obtaining

$$
\dot{\mathbf{A}}^{t} \mathbf{A}=\mathbf{W} .
$$

It follows that (65) entails no loss of generality insofar as the deformation, the dissipation or the Cauchy stress are concerned, provided that a rotation $\mathbf{A}(t)$ exists such that (68) is satisfied for any smooth $\mathbf{W}(t) \in \mathrm{Skw}$. To see that this is so, consider the initial-value problem

$$
\dot{\mathbf{B}}=\mathbf{W B} ; \quad \mathbf{B}(0)=\mathbf{B}_{0},
$$

where $\mathbf{B}_{0} \in$ Orth $^{+}$, for which standard theory yields the existence of a unique solution. As in [5] we define $\mathbf{Z}(t)=\mathbf{B B}{ }^{t}$ and obtain $\dot{\mathbf{Z}}=\mathbf{W}(t) \mathbf{Z}-\mathbf{Z W}(t)$ with $\mathbf{Z}(0)=\mathbf{I}$. The unique solution is $\mathbf{Z}(t)=\mathbf{I}$; therefore, $\mathbf{B}(t) \in$ Orth. To show that $\mathbf{B}(t) \in$ Orth $^{+}$, we compute $\dot{J}_{B}=$ $\mathbf{B}^{*} \cdot \dot{\mathbf{B}}=J_{B} \operatorname{tr} \mathbf{W}=0$, yielding $J_{B}(t)=J_{B}(0)=1$ and hence the claim. The desired rotation is then given by $\mathbf{A}=\mathbf{B}^{t}$. Thus, at each instant, a local reference $\kappa_{i}$ may be found to nullify the instantaneous plastic spin induced by the use of any alternative choice.

Remark The conventional theory of crystal plasticity [23-25] rests on a purely kinematical interpretation of plastic deformation according to which the rate of plastic deformation is presumed to be expressible in the form

$$
\dot{\mathbf{G}} \mathbf{G}^{-1}=\sum v_{i} \mathbf{s}_{i} \otimes \mathbf{n}_{i}
$$

in which the $v_{i}$ are the slips and the $\mathbf{s}_{i}$ and $\mathbf{n}_{i}$ are orthonormal vectors specifying the $i$ th slip system. The sum ranges over the currently active slip systems. This is clearly at odds with (65). To make contact between this hypothesis and the present work we choose $\dot{\mathbf{G}}_{1} \mathbf{G}_{1}^{-1}$ equal to the right-hand side of (70) and put

$$
\dot{\mathbf{G}}_{2} \mathbf{G}_{2}^{-1}=\mathbf{A}\left(\operatorname{Sym} \dot{\mathbf{G}}_{1} \mathbf{G}_{1}^{-1}\right) \mathbf{A}^{t},
$$

as required by (66) and (68). Defining $\overline{\mathbf{s}}_{i}=\mathbf{A} \mathbf{s}_{i}$ and $\overline{\mathbf{n}}_{i}=\mathbf{A} \mathbf{n}_{i}$, we obtain

$$
\dot{\mathbf{G}}_{2} \mathbf{G}_{2}^{-1}=\sum v_{i} \operatorname{Sym}\left(\overline{\mathbf{s}}_{i} \otimes \overline{\mathbf{n}}_{i}\right) .
$$

We assume the $\overline{\mathbf{s}}_{i}$ and $\overline{\mathbf{n}}_{i}$ to have vanishing material time derivatives in accordance the fact that slip-system vectors are fixed relative to the lattice vectors [23], which have vanishing material derivatives by hypothesis (cf. Sect. 2). The present model thus incorporates the conventional interpretation of plastic deformation provided that the $\mathbf{s}_{i}$ and $\mathbf{n}_{i}$ are permitted to be time-dependent. By convention, the latter are considered to be fixed in the standard interpretation and any change in slip-system orientation is attributed to the elastic deformation [23-25]. In the present framework we would regard solutions $\mathbf{G}_{2}$ to (72) as the plastic 
deformations while retaining the assumption of fixed slip-system vectors, here denoted by $\overline{\mathbf{s}}_{i}$ and $\overline{\mathbf{n}}_{i}$.

This discussion is intended for those wishing to interpret the present work in the context of the standard theory of crystal plasticity. However, unlike conventional crystal plasticity theory the present framework is entirely independent of hypotheses such as (70) pertaining to the representation of plastic deformation. In particular, we make no use of constitutive concepts from the standard theory. Instead, the present theory rests entirely on the continuum mechanics of anisotropic media.

\section{Plastic Flow}

Invoking well-known facts in convex analysis [8, 26, 27], we conclude from (60) and (62) that the current elastic range is a convex set and that $\dot{\mathbf{G}}\left(t_{a}\right) \mathbf{G}\left(t_{a}\right)^{-1}$ is directed along the exterior unit normal to the current yield surface at $\mathbf{S}\left(t_{a}\right)$, the latter being the limit as $\mid \mathbf{S}^{+}-$ $\mathbf{S}^{-} \mid \rightarrow 0$ of the normalized vector $\mathbf{S}^{+}-\mathbf{S}^{-} \in \mathcal{N}\left(\mathbf{S}\left(t_{a}\right)\right)$, where $H\left(\mathbf{S}^{+}\right)>0, H\left(\mathbf{S}^{-}\right)<0$, and $\mathcal{N}\left(\mathbf{S}\left(t_{a}\right)\right)$ is the normal space - the orthogonal complement of the tangent space- to the current yield surface at $\mathbf{S}\left(t_{a}\right)$. It follows that a scalar $\lambda(\mathbf{X}, t) \geq 0$ exists such that

$$
\dot{\mathbf{G}} \mathbf{G}^{-1}=\lambda F_{\mathbf{S}},
$$

wherein the partial derivative on the right-hand side is evaluated at fixed $\boldsymbol{\alpha}$ and for $\mathbf{S}$ such that $F(\mathbf{S}, \boldsymbol{\alpha})=0$. Thus, for plastic deformation to evolve $(\dot{\mathbf{G}} \neq \mathbf{0})$, it is necessary that $\lambda>0$. Inequality (64) then furnishes the restriction

$$
\mathbf{S} \cdot F_{\mathbf{S}}>0
$$

on the associated values of the arguments of the yield function. Integration of (73) yields the plastic deformation in principle, this being necessary for the determination of the evolving dislocation density via (2) and (21).

Because (60) is the first-order approximation to (56) for small elastic strain, it is likewise valid to first order in the stress given by (59). We are referring to the dimensionless stress obtained on dividing $\mathbf{S}$ by the largest eigenvalue of $\mathcal{C}$, the associated norm being on the order of that of the elastic strain. Accordingly, because (73) derives from (60), for consistency we approximate the derivative on the right-hand side of (73) to first order in stress and thus confine attention to yield functions that are quadratic in stress. An example is discussed in Sect. 7.

For (73) to qualify as a bona fide constitutive equation it must be invariant under compatible (i.e., differentiable) changes of reference placement and superposed rigid-body motions. Such invariance requires that $\lambda$ be likewise invariant. That this is so may be easily proved from the invariance of the function $F$, following, for example, the procedure given in [1]. Equation (73) must also be form-invariant under material symmetry transformations characterized by $\mathbf{H} \rightarrow \mathbf{H R}$ and $\mathbf{G} \rightarrow \mathbf{R}^{t} \mathbf{G}$, where $\mathbf{R}$ is any element of the (fixed, discrete) symmetry group intrinsic to the material, these leaving the Cauchy stress and deformation unchanged (cf. Sect. 2). This in turn requires that $\lambda \rightarrow \lambda$ under such transformations, this following from (13), (23), (61), (73) and the consequent transformation $F_{\mathbf{S}} \rightarrow \mathbf{R}^{t} F_{\mathbf{S}} \mathbf{R}$.

To determine $\lambda$ we assume that there exists an open interval of time during which plastic deformation evolves, this being necessary for the existence of a continuous, non-vanishing 
derivative $\dot{\mathbf{G}}$. Our constitutive hypotheses imply that $F$ vanishes identically in this interval, and hence the consistency condition

$$
F_{\mathbf{S}} \cdot \dot{\mathbf{S}}+F_{\alpha} \cdot \dot{\alpha}=0
$$

Here, we use (21) to derive

$$
\dot{\boldsymbol{\alpha}}=-\operatorname{tr}\left(\dot{\mathbf{G}} \mathbf{G}^{-1}\right) \boldsymbol{\alpha}+\left(\dot{\mathbf{G}} \mathbf{G}^{-1}\right) \boldsymbol{\alpha}+J_{G}^{-1} \mathbf{G}(\operatorname{Curl} \dot{\mathbf{G}}) .
$$

Substituting (73) yields an expression linear in $\lambda$ and its referential gradient, $\nabla \lambda$. Accordingly, (75) furnishes a linear constraint on $\dot{\mathbf{S}}$ and $\nabla \lambda$ jointly, which we do not pause to write explicitly.

For stresses belonging to the current yield surface, elastic unloading, and the concurrent cessation of plastic flow, is associated with stress increments that furnish a stress lying within the current elastic range. Accordingly, elastic unloading is identified by the conditions $H(\mathbf{S})=0, \lambda=0, F_{\mathbf{S}} \cdot \dot{\mathbf{S}}<0$. The alternative, referred to as plastic loading, is associated with the conditions $F(\mathbf{S}, \boldsymbol{\alpha})=0, \lambda>0$ and $F_{\mathbf{S}} \cdot \dot{\mathbf{S}} \geq 0$. Equation (75) applies in this case, and thus implies that $F_{\boldsymbol{\alpha}} \cdot \dot{\alpha} \leq 0$ during plastic loading. This subsumes neutral loading, defined by $F_{\mathbf{S}} \cdot \dot{\mathbf{S}}=0$ and $F_{\alpha} \cdot \dot{\alpha}=0$.

Suppose plastic evolution occurs in some region in $\kappa_{r}$. Suppose that an elastic-plastic boundary exists, separating the region in question from one in which plastic evolution has ceased. The field $\lambda$ vanishes on this boundary, furnishing Cauchy data for the consistency condition. It is possible to show that the coefficient of $\nabla \lambda$ in the consistency condition vanishes if and only $F_{\alpha} F_{\mathbf{S}} \in \mathrm{Sym}$. In such cases the condition reduces to a linear algebraic equation for $\lambda$.

\section{Residual Stress and Linear Elasticity Theory}

The present framework furnishes a basis for a model of linearly-elastic materials with residual stress, as well as a means to quantify residual stress in terms of the intrinsic properties of the material. Thus, to characterize the residual stress in the reference placement $\kappa_{r}$, we evaluate (5) at $\mathbf{F}=\mathbf{I}$, obtaining

$$
\mathbf{P}=\Pi_{0}(\mathbf{X})
$$

with

$$
\Pi_{0}(\mathbf{X})=J_{K_{0}}^{-1} \mathbf{K}_{0} \mathbf{S}_{0} \mathbf{K}_{0}^{t}
$$

where

$$
\mathbf{S}_{0}(\mathbf{X})=\frac{1}{2} \mathcal{C}\left[\mathbf{K}_{0}^{t} \mathbf{K}_{0}-\mathbf{I}\right]
$$

is the intrinsic stress.

Conventionally, the residual-stress problem is specified by requiring $\kappa_{r}$ to be an equilibrium placement under no body force with nil traction assigned on the entire boundary [28-30]. Thus,

$$
\operatorname{Div} \boldsymbol{\Pi}_{0}=\mathbf{0} \quad \text { in } \kappa_{r} \quad \text { and } \quad \boldsymbol{\Pi}_{0} \mathbf{N}=\mathbf{0} \quad \text { on } \partial \kappa_{r} .
$$

This represents an underdetermined system for the field $\mathbf{K}_{0}(\mathbf{X})$ and thus for the lattice-vector fields $\mathbf{r}_{i}^{0}(\mathbf{X})$. Accordingly, equilibrium considerations alone do not suffice to determine the 
residual stress. Further conditions must be given. For example, the prescription of Curl $\mathbf{K}_{0}^{-1}$ has yielded definite results in some circumstances [31]. In principle, the field $\mathbf{K}_{0}(\mathbf{X})$ should be viewed as an element of an equilibrium state, if any, of the dynamical system that describes the evolution of the body. This field is then obtained by identifying $\kappa_{r}$ with the associated equilibrium placement. Consistency with this view requires that

$$
F\left(\mathbf{S}_{0}, \boldsymbol{\alpha}_{0}\right)<0
$$

at all $\mathbf{X}$ in $\kappa_{r}$, where $\boldsymbol{\alpha}_{0}$ follows from $\mathbf{K}_{0}$ via (21).

Consider a displacement field $\mathbf{u}(\mathbf{X}, t)$ superposed on $\kappa_{r}$. The induced deformation gradient and strain are given respectively by

$$
\mathbf{F}=\mathbf{I}+\nabla \mathbf{u}
$$

and

$$
\boldsymbol{\epsilon}=\boldsymbol{\varepsilon}+\frac{1}{2}(\nabla \mathbf{u})^{t}(\nabla \mathbf{u}), \quad \text { where } \boldsymbol{\varepsilon}=\operatorname{Sym}(\nabla \mathbf{u}) .
$$

The associated elastic strain is

$$
\mathbf{E}=\frac{1}{2}\left(\mathbf{K}_{0}^{t} \mathbf{K}_{0}-\mathbf{I}\right)+\mathbf{K}_{0}^{t} \boldsymbol{\epsilon} \mathbf{K}_{0}
$$

and we assume $\mathbf{S}=\mathcal{C}[\mathbf{E}]$ to be such that $F\left(\mathbf{S}, \boldsymbol{\alpha}_{0}\right)<0$. Accordingly, (5), (6) and (59) may be combined to obtain the linear approximation [28-30]

$$
\mathbf{P}=\Pi_{0}(\mathbf{X})+(\nabla \mathbf{u}) \boldsymbol{\Pi}_{0}(\mathbf{X})+\mathcal{L}(\mathbf{X})[\varepsilon]+o(|\nabla \mathbf{u}|),
$$

where $\mathcal{L}$ is defined by

$$
\mathcal{L}(\mathbf{X})[\mathbf{A}]=J_{K_{0}}^{-1} \mathbf{K}_{0}\left\{\mathcal{C}\left[\mathbf{K}_{0}^{t} \mathbf{A} \mathbf{K}_{0}\right]\right\} \mathbf{K}_{0}^{t}
$$

for all $\mathbf{A} \in$ Sym. These results render explicit the roles played by the intrinsic material properties $\mathcal{C}$ as well as the plastic deformations $\mathbf{K}_{0}(\mathbf{X})$ admitted by (78)-(80).

\section{Example: Cubic Crystals}

For cubic crystals the strain-energy function $W$ and the yield function $F$ are invariant under the group consisting of the rotations that map a cube to itself. To specify $W$ (cf. (59)) we require the invariant functions that are homogeneous of degree two in the elastic strain $\mathbf{E}$, these being common to each of the five subclasses of cubic symmetry. They are [32]

$$
(\operatorname{tr} \mathbf{E})^{2}, \quad E_{11} E_{22}+E_{11} E_{33}+E_{22} E_{33} \text { and } E_{12}^{2}+E_{13}^{2}+E_{23}^{2},
$$

where $E_{i j}=\mathbf{E} \cdot \operatorname{Sym}\left(\mathbf{e}_{i} \otimes \mathbf{e}_{j}\right)$ and $\left\{\mathbf{e}_{i}\right\}$ is an orthonormal basis aligned with the cube axes (i.e.; with the elements of the set $\left\{\mathbf{I}_{i}\right\}$ of lattice vectors, or their duals). We emphasize the fact that the orthonormal basis, and hence the orientation of the cube, is arbitrary. Once chosen, it is considered to remain fixed at the material point in question, and, for materially-uniform bodies, carried over to all such points. The choice does, however, affect $\mathbf{K}_{0}(\mathbf{X})$ (cf. Sect. 2), this being a natural consequence of the fact that $\left\{\mathbf{I}_{i}\right\}$ is involved in the definition of plastic deformation. These points are significant for the practical implementation of the theory. 
To elaborate, consider the set $\left\{\mathbf{I}_{i}^{*}\right\}$ of lattice vectors given by $\mathbf{l}_{i}^{*}=\mathbf{A} \mathbf{l}_{i}$, where $\mathbf{A}$ is a fixed, spatially uniform rotation. Then, $\mathbf{l}^{* i}=\mathbf{A} \mathbf{l}^{i}$ and the plastic deformation based on the starred lattice is (cf. Sect. 2) $\mathbf{G}^{*}=\mathbf{A G}$. This satisfies the flow rule (cf. (73))

$$
\dot{\mathbf{G}}^{*}\left(\mathbf{G}^{*}\right)^{-1}=\lambda \mathbf{A}\left(F_{\mathbf{S}}\right) \mathbf{A}^{t}=\lambda F_{\mathbf{S}^{*}}^{*},
$$

where $\mathbf{S}^{*}=\mathbf{A S A}^{t}$ is the stress relative to the starred lattice and $F^{*}\left(\mathbf{S}^{*}, \boldsymbol{\alpha}^{*}\right)=F(\mathbf{S}, \boldsymbol{\alpha})$, with $\boldsymbol{\alpha}^{*}=\mathbf{A} \boldsymbol{\alpha} \mathbf{A}^{t}$, is the associated yield function. It follows that if $\mathbf{G}(t)$ is a plastic deformation with $\mathbf{G}\left(t_{0}\right)=\mathbf{G}_{0}$, then $\mathbf{G}^{*}$ is the same plastic deformation, modulo rotation, provided that $\mathbf{G}^{*}\left(t_{0}\right)=\mathbf{A G}_{0}$. The sole effect of an alternative choice of lattice is to alter the initial value of plastic deformation by a uniform rotation. Given the field $\left\{\mathbf{r}_{i}^{0}(\mathbf{X})\right\}$, the specification of the initial plastic deformation is thus tantamount to a selection of lattice vectors.

Returning to the discussion of cubic symmetry, some simplification is achieved by using the deviatoric strain $\overline{\mathbf{E}}=\operatorname{Dev} \mathbf{E}$. This yields

$$
E_{11} E_{22}+E_{11} E_{33}+E_{22} E_{33}=\frac{1}{3}(\operatorname{tr} \mathbf{E})^{2}-\left(\bar{E}_{11}^{2}+\bar{E}_{22}^{2}+\bar{E}_{33}^{2}\right)
$$

and so the strain-energy function is expressible in the form [33]

$$
\tilde{W}(\mathbf{E})=\frac{1}{2}\left[C_{1}(\operatorname{tr} \mathbf{E})^{2}+C_{2}\left(\bar{E}_{11}^{2}+\bar{E}_{22}^{2}+\bar{E}_{33}^{2}\right)\right]+C_{3}\left(E_{12}^{2}+E_{13}^{2}+E_{23}^{2}\right),
$$

where $C_{i}$ are the (constant) moduli, giving the strain energy as a linear combination of three independent quadratic forms. Because each is positive definite, the relevant constitutive hypothesis is met if and only if each $C_{i}>0$. The associated stress, obtained by equating $\tilde{\mathbf{S}}(\mathbf{E}) \cdot \dot{\mathbf{E}}$ to $(\tilde{W}(\mathbf{E}))$, is

$$
\begin{aligned}
\tilde{\mathbf{S}}(\mathbf{E})= & C_{1}(\operatorname{tr} \mathbf{E}) \mathbf{I}+C_{2}\left(\bar{E}_{11} \mathbf{e}_{1} \otimes \mathbf{e}_{1}+\bar{E}_{22} \mathbf{e}_{2} \otimes \mathbf{e}_{2}+\bar{E}_{33} \mathbf{e}_{3} \otimes \mathbf{e}_{3}\right) \\
& +C_{3}\left[E_{12}\left(\mathbf{e}_{1} \otimes \mathbf{e}_{2}+\mathbf{e}_{2} \otimes \mathbf{e}_{1}\right)+E_{13}\left(\mathbf{e}_{1} \otimes \mathbf{e}_{3}+\mathbf{e}_{3} \otimes \mathbf{e}_{1}\right)\right. \\
& \left.+E_{23}\left(\mathbf{e}_{2} \otimes \mathbf{e}_{3}+\mathbf{e}_{3} \otimes \mathbf{e}_{2}\right)\right] .
\end{aligned}
$$

Turning to the yield function, we simplify matters by assuming that its dependence on stress and dislocation density can be decoupled. Mainly for illustrative purposes, we also adopt the widespread assumption that pressure, proportional to $\operatorname{tr} \mathbf{T}$, has negligible effect on yield, this effectively limiting the applicability of the model to circumstances in which the pressure is not too large. To examine the implications, we use (10) and (11) to obtain $\operatorname{tr} \mathbf{T}=$ $J_{H}^{-1} \operatorname{tr}(\mathbf{C S})$; Equations (20) and (59) then furnish $\operatorname{tr} \mathbf{T}=\operatorname{tr} \mathbf{S}+o(|\mathbf{E}|)$. Consistency with our previous assumptions thus implies that the current yield function $H(\cdot)=F(\cdot, \boldsymbol{\alpha})$ should depend on $\mathbf{S}$ via $\overline{\mathbf{S}}=\operatorname{Dev} \mathbf{S}$; we write $H(\mathbf{S})=\bar{F}(\overline{\mathbf{S}})$. To represent this function we require the scalar invariants of $\overline{\mathbf{S}}$ under the cubic symmetry group up to quadratic order (cf. Sect. 5). The only linear invariant, $\operatorname{tr} \overline{\mathbf{S}}$, vanishes identically, leading to a function homogeneous of degree two:

$$
\bar{F}(\overline{\mathbf{S}})=\frac{1}{2} A_{1}\left(\bar{S}_{11}^{2}+\bar{S}_{22}^{2}+\bar{S}_{33}^{2}\right)+A_{2}\left(S_{12}^{2}+S_{13}^{2}+S_{23}^{2}\right),
$$

where $A_{1,2}$ are constants. Then,

$$
\begin{aligned}
F_{\mathbf{S}}= & A_{1}\left(\bar{S}_{11} \mathbf{e}_{1} \otimes \mathbf{e}_{1}+\bar{S}_{22} \mathbf{e}_{2} \otimes \mathbf{e}_{2}+\bar{S}_{33} \mathbf{e}_{3} \otimes \mathbf{e}_{3}\right) \\
& +A_{2}\left[S_{12}\left(\mathbf{e}_{1} \otimes \mathbf{e}_{2}+\mathbf{e}_{2} \otimes \mathbf{e}_{1}\right)+S_{13}\left(\mathbf{e}_{1} \otimes \mathbf{e}_{3}+\mathbf{e}_{3} \otimes \mathbf{e}_{1}\right)\right. \\
& \left.+S_{23}\left(\mathbf{e}_{2} \otimes \mathbf{e}_{3}+\mathbf{e}_{3} \otimes \mathbf{e}_{2}\right)\right],
\end{aligned}
$$


and we obtain

$$
\mathbf{S} \cdot F_{\mathbf{S}}=A_{1}|\overline{\mathbf{S}}|^{2}+2\left(A_{2}-A_{1}\right)\left(S_{12}^{2}+S_{13}^{2}+S_{23}^{2}\right) .
$$

Accordingly, (74) is satisfied if and only if $\overline{\mathbf{S}} \neq \mathbf{0}$ and

$$
A_{1}>0, \quad A_{2}>A_{1} \text {. }
$$

We observe, from (73) and (93), that $\operatorname{tr} \dot{\mathbf{G}} \mathbf{G}^{-1}=0$ and thus that $J_{G}=J_{G_{0}}$. An expression for the yield function equivalent to (92) is given in [34].

It follows from (95) that $\bar{F}(\cdot)$ is positive definite. To allow for yielding in a manner consistent with our assumptions, it is thus necessary that the yield function be expressible in the form

$$
F(\mathbf{S}, \boldsymbol{\alpha})=\bar{F}(\overline{\mathbf{S}})-K(\boldsymbol{\alpha}),
$$

where $K(\cdot)$ is positively valued function subject to the material-symmetry restriction $K(\boldsymbol{\alpha})=K\left(\mathbf{R}^{t} \boldsymbol{\alpha} \mathbf{R}\right)$. The form that this function should take in applications to real crystals remains open, although it is possible to advance some proposals on the basis of current ideas on the subject of scale dependence [35]. One, compatible with cubic symmetry, is

$$
K=\left(k_{0}+k_{1}|\boldsymbol{\alpha}|^{p}\right)^{2},
$$

where $p, k_{0,1}$ are positive constants, $\sqrt{K}$ then being proportional to the yield stress associated with a pair of shear tractions exerted on opposing faces of a cube and directed parallel to its axes. This expression models the hardening of the crystal due to the presence of dislocations [12]. However, we note that the development of a predictive theory of strain hardening has yet to be achieved, this shortcoming arguably posing the main open problem in the subject.

Acknowledgements This work was performed under the auspices of the US Department of Energy by the Lawrence Livermore National Laboratory, under contract no. W-7405-Eng-48. Part of this work was initiated during the tenure of a Lawrence Scholarship held by A. Gupta from 2006 through 2008 in support of graduate studies at the University of California.

Open Access This article is distributed under the terms of the Creative Commons Attribution Noncommercial License which permits any noncommercial use, distribution, and reproduction in any medium, provided the original author(s) and source are credited.

\section{References}

1. Gupta, A., Steigmann, D.J., Stölken, J.S.: On the evolution of plasticity and incompatibility. Math. Mech. Solids 12, 583-610 (2007)

2. Noll, W.: Materially uniform simple bodies with inhomogeneities. Arch. Ration. Mech. Anal. 27, 1-32 (1967)

3. Wang, C.-C.: Mathematical Principles of Mechanics and Electromagnetism, Part A: Analytical and Continuum Mechanics. Plenum Press, New York (1979) (Chapter 4)

4. Epstein, M., Elzanowski, M.: Material Inhomogeneities and Their Evolution. Springer, Berlin (2007)

5. Gurtin, M.E.: An Introduction to Continuum Mechanics. Academic Press, Orlando (1981)

6. Il'iushin, A.A.: On the postulate of plasticity. Prikl. Mat. Mekh. 25, 746-752 (1961)

7. Naghdi, P.M., Trapp, J.A.: On the nature of normality of plastic strain rate and convexity of yield surfaces in plasticity. ASME J. Appl. Mech. 42, 61-66 (1975)

8. Lucchesi, M., Podio-Guidugli, P.: Materials with elastic range: A theory with a view towards applications, Part II. Arch. Ration. Mech. Anal. 110, 9-42 (1990) 
9. Lucchesi, M., Silhavy, M.: Il'yushin's conditions in non-isothermal plasticity. Arch. Ration. Mech. Anal. 113, 121-163 (1991)

10. Truesdell, C., Noll, W.: The Nonlinear Field Theories of Mechanics. Handbuch der Physik, vol. 3. Springer, Heidelberg (1965)

11. Cullity, B.: Elements of X-Ray Diffraction. Addison-Wesley, Reading (1978)

12. Taylor, G.I.: The mechanism of the plastic deformation of crystals. Part I: Theoretical. Proc. R. Soc. Lond. A 145, 326-387 (1934)

13. Davini, C.: Elastic invariants in crystal theory. In: Ball, J.M. (ed.) Material Instabilities in Continuum Mechanics and Related Mathematical Problems, pp. 85-105. Oxford University Press, Oxford (1988)

14. Cermelli, P., Gurtin, M.E.: On the characterization of geometrically necessary dislocations in finite plasticity. J. Mech. Phys. Solids 49, 1539-1568 (2001)

15. Arsenlis, A., Parks, D.M.: Crystallographic aspects of geometrically-necessary and statistically-stored dislocation density. Acta Mater. 47, 1597-1611 (1999)

16. Cleja-Tigoiu, S., Soos, E.: Elastoviscoplastic models with relaxed configurations and internal variables. Appl. Mech. Rev. 43, 131-151 (1990)

17. Cleja-Tigiou, S.: Consequences of the dissipative restrictions in finite anisotropic elasto-plasticity. Int. J. Plast. 19, 1917-1964 (2003)

18. Stölken, J.S., Evans, A.G.: A microbend test method for measuring the plasticity length scale. Acta Mater. 46, 5109-5115 (1998)

19. Coleman, B.D., Hodgdon, M.L.: On shear bands in ductile materials. Arch. Ration. Mech. Anal. 90, 219-247 (1985)

20. Gao, X.-L.: Strain gradient plasticity solution for an internally pressurized thick-walled cylinder of an elastic linear-hardening material. Z. Angew. Math. Phys. 58, 161-173 (2007)

21. Liu, I.-S.: Continuum Mechanics. Springer, Berlin (2002)

22. Abeyaratne, R., Knowles, J.K.: On the driving traction acting on a surface of strain discontinuity in a continuum. J. Mech. Phys. Solids 38, 345-360 (1990)

23. Havner, K.S.: Finite Plastic Deformation of Crystalline Solids. Cambridge University Press, Cambridge (1992)

24. Rengarajan, G., Rajagopal, K.: On the form for the plastic velocity gradient $L_{p}$ in crystal plasticity. Math. Mech. Solids 6, 471-480 (2001)

25. Gurtin, M.E., Fried, E., Anand, L.: Mechanics and Thermodynamics of Continua. Cambridge University Press, Cambridge (2010)

26. van Tiel, J.: Convex Analysis. Wiley, Chichester (1984)

27. Lubliner, J.: Plasticity Theory. Dover, New York (2008)

28. Hoger, A.: On the determination of residual stress in an elastic body. J. Elast. 16, 303-324 (1986)

29. Man, C.-S., Carlson, D.: On the traction problem of dead loading in linear elasticity with residual stress. Arch. Ration. Mech. Anal. 128, 223-247 (1994)

30. Paroni, R., Tomassetti, G.: A variational justification of linear elasticity with residual stress. J. Elast. 97, 189-206 (2009)

31. Sfyris, D., Charalambakis, N., Kalpakides, V.K.: Continuously dislocated elastic bodies with a neoHookean like energy subjected to anti-plane shear. J. Elast. 93, 245-262 (2008)

32. Green, A.E., Adkins, J.E.: Large Elastic Deformations. Oxford University Press, Oxford (1970)

33. Ericksen, J.L.: Constitutive theory for some constrained elastic crystals. Int. J. Solids Struct. 22, 951-964 (1986)

34. Cazacu, O., Barlat, F.: Application of the theory of representation to describe yielding of anisotropic aluminum alloys. Int. J. Eng. Sci. 41, 1367-1385 (2003)

35. Kubin, L.P., Mortensen, A.: Geometrically necessary dislocations and strain-gradient plasticity: A few critical issues. Scr. Mater. 48, 119-125 (2003) 